\title{
An Efficient Technique for Object Recognition from a Complex Image
}

\author{
Gurjinder Singh $^{1}$, Jaspreet Kaur ${ }^{2}$ \\ ${ }^{1,2}$ Guru Gobind Singh College of Science and Engineering Guru Kashi University Talwandi Sabo (Bathinda), India
}

\begin{abstract}
Valuable information can be hidden in images, however, few research discuss data mining on them. Image retrieval means searching, browsing and retrieving images from image databases. There are two different methodologies for image retrieval i.e. text based image retrieval and content based image retrieval. Former one is obsolete. In latter one many visual features like texture, size, intensities, and frequency of pixels and color of image are extracted. In query-by-example search extracted featured are compared with stored ones. In this work an efficient for extracting image features is considered using intensity histogram of gray color image. Here in this general framework based on the decision tree for mining and processing image data. Pixel wised image features were extracted and transformed into a database-like table which allows various data mining algorithms to make explorations on it. Finally results of average gradient vectors are to be compared with previously stored one dimensional array of intensities to find similarities in image data.
\end{abstract}

Keywords: Convo-Detect, Hybrid Projection, EOAC, DC, EDH, DAC ImgDataRet, Hamming20, Keller6, Brock20

\section{Image Extraction}

Data Mining is termed as discovering hidden values in data warehouse, extracting hidden information from huge databases is a powerful new methodology which has helped many organizations nowadays to focus on significant information in their data warehouses. [1] Data mining techniques can be implemented rapidly on various platforms on high performance parallel processing computers or client/servers. Data mining tools can work with heavy databases to find answers to various business queries. [3] Many data mining operations are managed outside of data warehouse nowadays and thus require extra steps for extraction, import and to analyze preprocessed data. Also, when new things require operational data implementation, collaborations with the warehouse improves the application of outcomes from data mining. The resulting analytic data warehouse can be applied to improve business processes throughout the organization, in areas such as substantial degree of relevance management, fraud detection, new product rollout, and so on. The starting point of data mining process is a data warehouse having collaboration of its internal data with external market data from web about rival companies competing with them.[1,2] Previous raw information related to potential customers provides excellent basis for analyst software modules. An OLAP server favors more advanced flexible and fast data access to be applied when navigating data in some data mapping scheme for standardization. The data mining access server must be integrated with OLAP server and data warehouse. It is the job of data mining access server to advance process centric data that will give major data mining objectives like prospecting, promotional integration and campaign management.[3] Integration of newly arrived metadata with existing data warehouse allows operational decisions to be directly focused and tracked. As a result when data warehouse grows with newly bindings in decisions, organization can mine continuously best results to be practiced for future decision making.

\subsection{Image Mining Process}

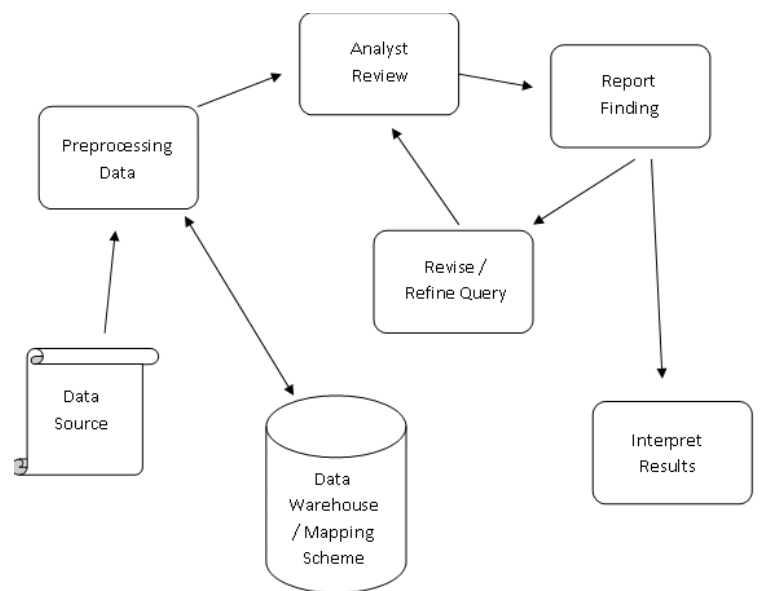

Figure 1: Data mining process interpreting mined results.

Data mining is the feature detection technique of image data and the use of software based techniques for finding image patterns and bitmap regularities in sets of image data. The computer program is responsible for observing the image bit patterns by elucidating the pre-defined rules and image features in the image data. Data collected from different sources like databases or flat files or any other resources like web is made to be preprocessed. Various standardization techniques of data cleaning or formatting are to be used by data warehouse or mapping schemes. [4] Further preprocessed data is made to be reviewed by data analyst who is done nowadays by computers software. In earlier days it was manually done by data analysts. After reviewing preprocessed data analyst reports are to be made which is revised or refined if something is needed with hand on information to generate finally mined or interpreted results.

\subsection{Spatial Relations}

One of the most mandatory features in many multimedia documents is management of spatial coherence. Management of spatial relation of components of image data is done by considering its rectangular Cartesian coordinates. 


\section{International Journal of Science and Research (IJSR) \\ ISSN (Online): 2319-7064}

Index Copernicus Value (2015): 78.96 | Impact Factor (2015): 6.391

The spatial axis position of a component in object in image data is represented by rectangular coordinates and the relations between components are calculated mathematically. [5,3] A multimedia document which is composed of images, flowcharts, and other random graphics as well as image text is another example that proves the management of spatial coordinates for layout information in an image [6]. In other applications of image processing such as geographical information system (GIS), the image pattern representation and random indexing of abstract and finite spatial relations in some regions of image is studied. A 2D stream of bits [3], [5] is an image data indexing technique for representing a spatial pattern between different components of an image; 2D stream of bits represents few patterns in position of components in neighboring regions, which is composed of horizontal and vertical ordering of image components. Also, it gives several distinct levels of a sparse-strict spatial relation, where the stiffness of directional data differs from one level of hierarchy to other. In [8] a set of two dimensional bits such as ' 00 ' ' 11 ' ' 01 ' ' 10 ' ' 111 ' ' 100 ' ' 101 ' ' 001 ' and ' 011 ' is defined as primary relations for representing spatial region of image data coordinates in observed components of image. This method along with 2D stream of bits is suitable for sparse bit evaluation of spatial relation in image patterns. The advantage of above method of representation of bits of image is that it left subtle denominations of spatial relations that are not required to be evaluated. However, the spatial relation is not distance-dependent, non-interval-based bits in image data are out of their scope of findings.

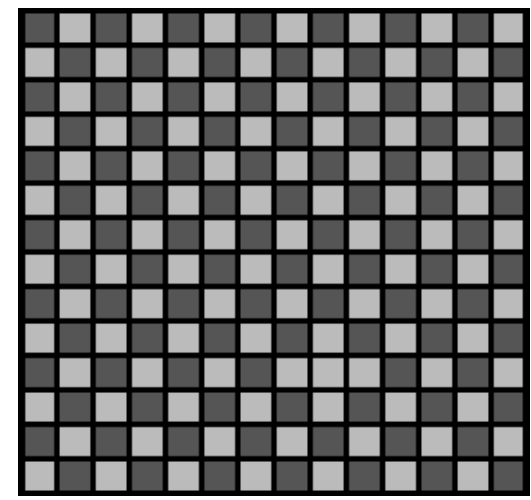

Figure 2: Check-board with one square colored incorrectly

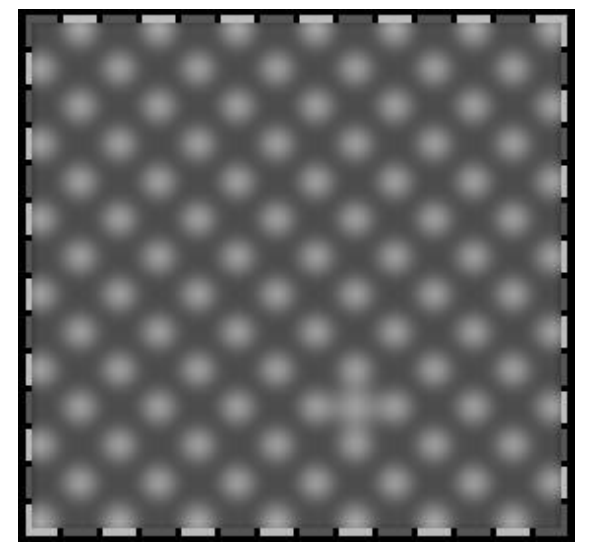

Figure 3: Blur image of check-board

\subsection{Temporal Relations}

A recent study depends upon bit representation and image data management of temporal relation considers video based applications of image processing such as video information databases (VID). There are two basic techniques for representing time based relationship between objects in multimedia data of a moving image: One is a point-based image data representation containing time lag of various colors in a multimedia document, and the other is an interval-based image data representation which contains intervals of data shifts from neighboring regions of data. [7, $11,12]$ The point based representation contains the Cartesian position of objects by points in the image data on timeline, whereas the interval-based representation contains the cohesion of image objects by means of their intervals of the occurrences of similar intensities in neighboring regions of an image. Many studies shows.

Interval based modeling technique of data representation. is used to manage the time lag in between various component videos objects that are used in multimedia document. Video elements of document (VID) are shown as if a stream of video bit patterns being defined by the time lag intervals, a video object comprises of different streams of video frames which are stored in contiguous manner. $[14,15,16]$ Video objects can be changed using two operations of merge and overlap. To be part of the definition of every video object the textual annotation of the contents of the video are merged or overlapped. Both the frame based and interval based specification are used to query a video object in OVID. Gibbs et.al [13] used a timed based representation of component objects. Sequential representation of component objects is used in this study. So this technique did not give a natural representation of a condition. Reverse and temporal relations were discussed and extended by little and Ghafoor. Allen's basic definition of temporal relations was followed by Hopner.

\subsection{Image Extraction Through Mining}

The technique of handling the association of image data and its patterns which are not stored in its images is known as image mining. Different methods of image processing and retrieval are used by this technique. There are two approaches to mining, firstly to mine from a large database of images and the other one performed on different collections of images and the data. The co-ordination of structures and the human brain can be deteriorated by this technique. The actual meaning of image mining is the production of different patterns with no knowledge of the image content. There can be different kinds of patterns like description, temporal, spatial etc. [17, 15] All huge image databases are being handled by the image mining.

\subsection{Content Based Retrieval Technique}

In this technique a complete image is segmented into small parts which further comprise to form the group of small images based on the index and layout feature. For the large scale data management (AIR) 


\section{International Journal of Science and Research (IJSR) \\ ISSN (Online): 2319-7064}

Index Copernicus Value (2015): 78.96 | Impact Factor (2015): 6.391

Automated Image Retrieval System is used to develop the Image Science and machine Vision (ISMV) of ORNL. In the olden days textual description was handled manually. But there were different loopholes such as semantic structure can vary with respect to a retrieval task and it was also a very time consuming process with regard to the size of image databases. The solution to these problems was content descriptors. In these technique global dimensions of the image was used. [20, 12, 19] Due to this another concept came into being that was named as Image segmentation in which the internal content of image was sidelined .But there is no correct technique of segmentation. Although there are different techniques but we could not arrive at the best one. So a definition of segmentation came into being i.e. it is a process with the help of which we can label each and every point of image including the class label. Another feature of segmentation is the capability to work in the unplanned sequence. These techniques do not rely on continuous human efforts. This problem is due to the restriction applied on the size. Due to everyday increase in the size of image which leads to hindrance in the way of conversation. [7, 9, $10,11]$.

\subsection{Image Object Retrieval Using Context Aware Technique}

The object recognition can be improved with the use of a knowledge base. This process can be achieved by going through two stages, first one is to define the properties of each segmented region and the second one is to classify results based on the views of the classified objects. The scene contextual information is based on the height, sensory information, the analysis of the visibility mapping etc. the methodology of object recognition is performed at two databases i.e. multi view digital angular camera and multi angular worldview. [18] The gap between the low level and high level interpretation can be decreased with the help of image segmentation. The low level image content should be user friendly so that changes can be performed at any stage in a image in CBIR system.

\section{Flow Chart For Proposed Techniques}

Firstly image is made input through standard image input construct imread(). Then its preprocessing is done by observing differently labeled regions of intensities. Normalization of regions is done through standard method i.e. select strongest(). This will return twenty strongest object particles out of hundred found object particles. Then detection of image object particles is done with Sobel and Minkowsky detection methods. Their result is handed over to Harris Feature Detection Map, that will return two dimensional intensity metric of image object particles.

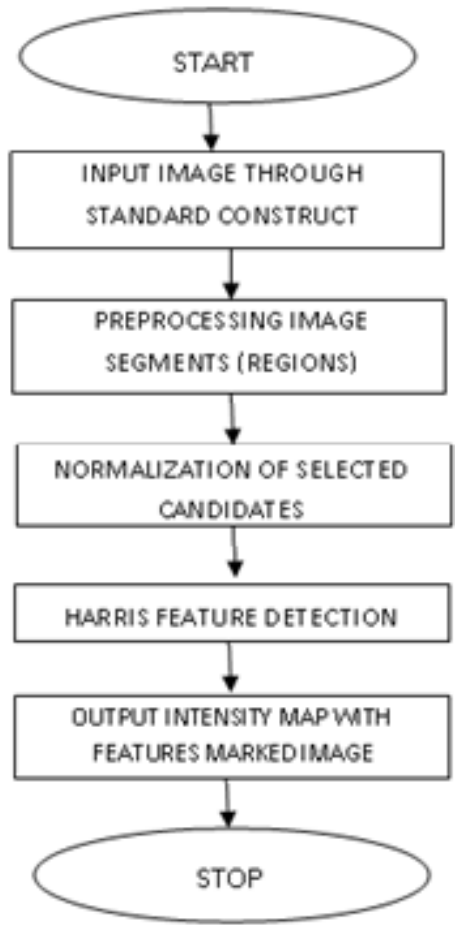

Figure: Flow chart of Image Retrieval Process

\section{Tools Used}

Here in this work, Computer Vision toolbox of Matlab 2013 is used. It includes various features of processing images through standard techniques. These techniques are recently developed by Matlab developers. Computer Vision System Toolbox provides algorithms and tools for the design and simulation of computer vision and video processing systems. The toolbox includes algorithms for feature extraction, motion detection, object detection, object tracking, stereo vision, video processing, and video analysis. Tools include video file I/O, video display, drawing graphics, and compositing. Capabilities are provided as MATLAB functions, MATLAB System objects, and Simulink blocks. For rapid prototyping and embedded system design, the system toolbox supports fixed-point arithmetic and C-code generation. Its Key Features includes; Feature detection, including FAST, Harris, Shi \& Tomasi, SURF, and MSER detectors Feature extraction and putative feature matching, Object detection and tracking, including Viola-Jones detection and CAM Shift tracking, Motion estimation, including block matching, optical flow, and template matching, RANSAC-based estimation of geometric transformations or fundamental matrices, Video processing, video file I/O, video display, graphic overlays, and compositing, Block library for use in Simulink

\section{Results and Discussions}

\subsection{Generation of Corner Matrix in MATLAB}

The implementation of the ImgDataRet algorithm using Computer Vision Toolbox in MATLAB. The implementation was done in 4 phases:-

1) Definition of Inputs and Outputs

2) Creating the Spatial-Region using view Computer Vision tool 


\section{International Journal of Science and Research (IJSR) \\ ISSN (Online): 2319-7064 \\ Index Copernicus Value (2015): 78.96 | Impact Factor (2015): 6.391}

3) Defining type and size of input image

4) Simulation of result by finding different object particles from each image.

The following are some snapshots of the inputs, inference engine and output on the editor:-

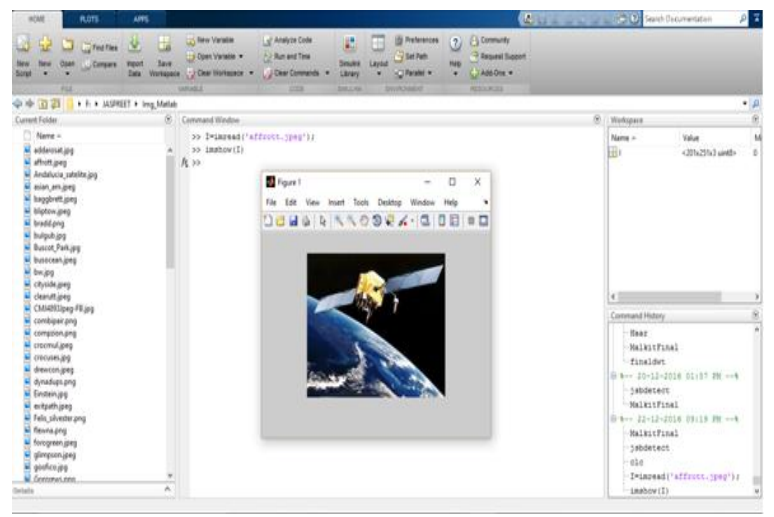

Figure 5: Variable I for input image

\subsection{Designing Interface for Object Particles Retrieval (ImgDataRet)}

Interface for simulating ImgDataRet method contains one input parameter that is an Image. One input vector is jpeg image or any other dot extension. Another three are output parameters represented with different push buttons in this interface. One output parameter gives CPU elapsed time for finding Maximum Object Particles using ImgDataRet algorithm. Second output parameter gives status of each particle that each particle is a part of some bounding region. While third output parameter gives extracted features found in the input Image in the form of two dimensional Matrix containing two vectors corresponding to object particles.

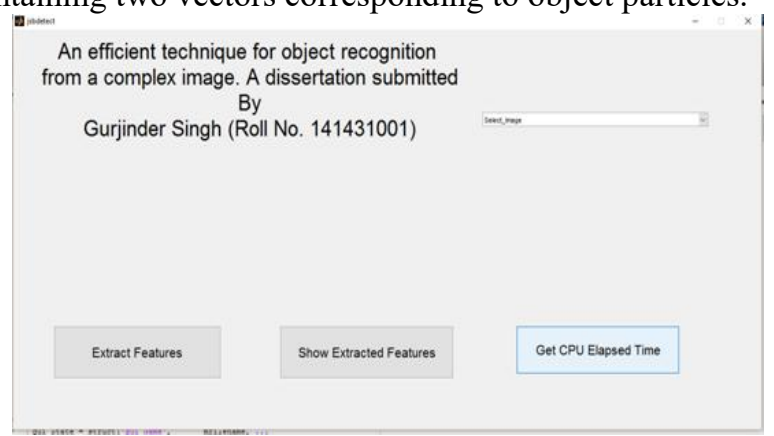

Figure 6: Fig File of Interface Design

\subsection{Implementation of ImgDataRet Algorithm}

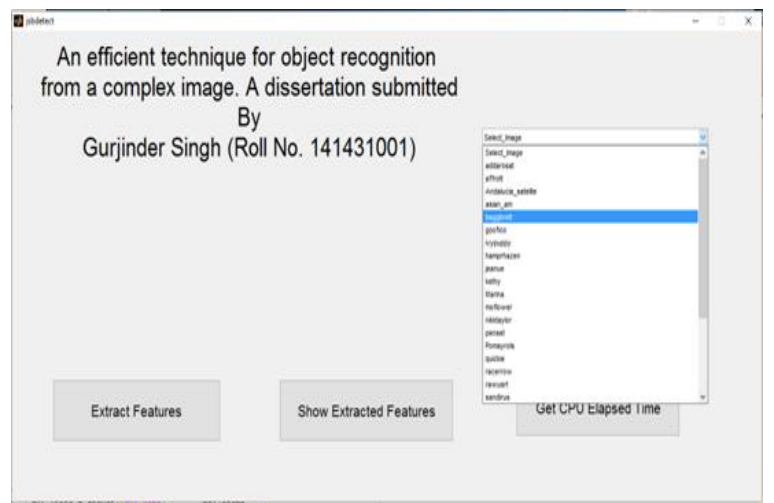

Figure 7: Interface of ImgDataRet Approach

\subsection{After Object Particles Extraction}

Extraction of image object particles from input image done by first applying Harris Edge Detection Algorithm. Then results of object particles are normalized with Distance Auto Correlation unit transformations. Object particles with green cross points are drawn here in the output image.

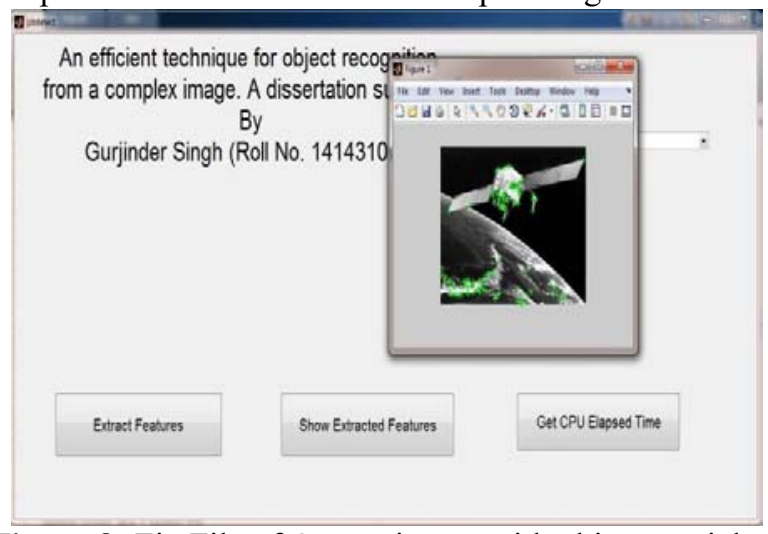

Figure 8: Fig File of Output image with object particles.

\subsection{Extracted Object Particles Map}

Object particles from an image represent severe intensity regions which are vulnerable to noise, due to which Distance Auto Correlation with noise intensities may distort the image. Image Object particles are key points through which noise can find coherence and image objects are residing in those regions where objects particles are there.

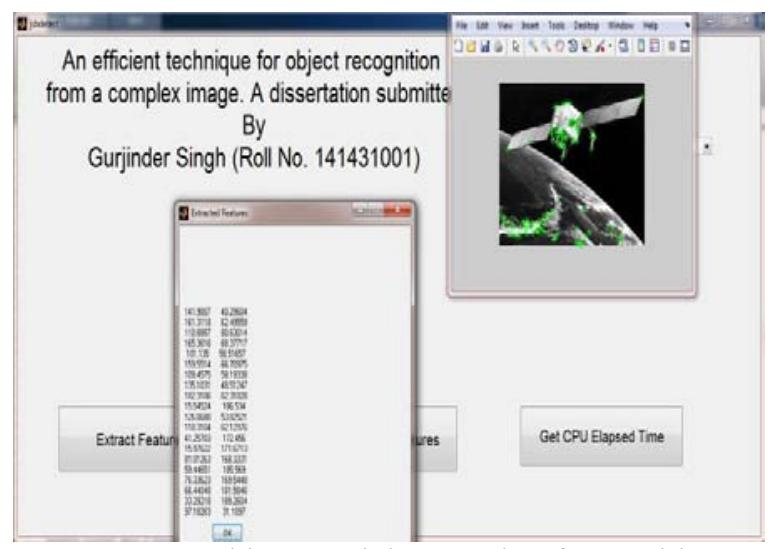

Figure 9: Object Particles Matrix of Intensities

\subsection{Extracted CPU-Time}

Total cpu elapsed time is the aggregation of number of cpu cycles needed in finding distance correlation vector and mapping intensities with object particles. Here in this interface a push button for extracting object particles map i.e. $2 \times 1$ Matrix of object particles is there. Another push button is provided for getting cpu elapsed time of ImgDataRet Algorithm. 


\section{International Journal of Science and Research (IJSR) \\ ISSN (Online): 2319-7064}

Index Copernicus Value (2015): 78.96 | Impact Factor (2015): 6.391

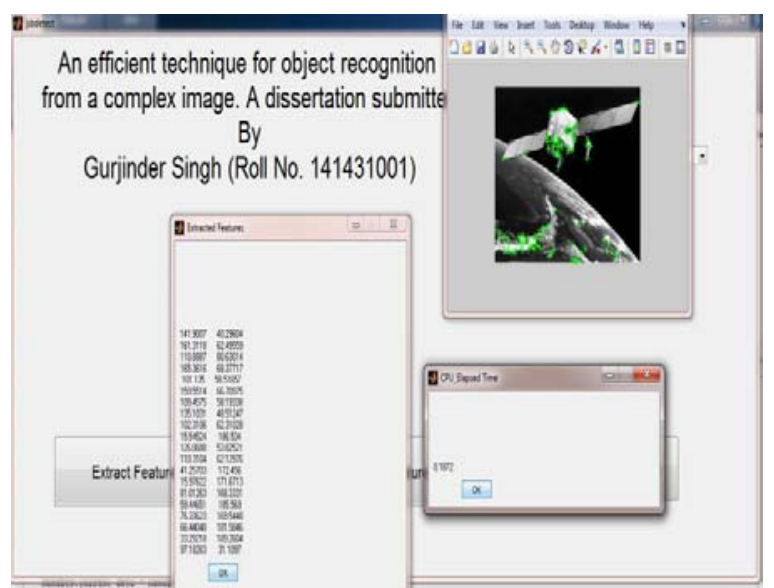

Figure 10: CPU elapsed time of ImgDataRet.

Standard images of University of Ottawa benchmark are tested on various levels of machines. Their variable object particles depend upon complexity of the image. For example Satelite1 is a heavily loaded image with image object particles of number 100. Since the number of edges are independent on number of object particles but their coherence depends on activities or complexity in an image.

Table 1: Read and Run Times of Standard images

\begin{tabular}{|c|c|c|}
\hline Image Name (.jpeg) & Read_Time (ms) & Run_Time (ms) \\
\hline addarosat & 0.05 & 26.56 \\
\hline affrott & 0.18 & 629.72 \\
\hline andalucia_satelite & 0.72 & 411.89 \\
\hline baggbrett & 0.05 & 20.35 \\
\hline busocean & 0.25 & 408.46 \\
\hline Jeanue & 0.08 & 5.36 \\
\hline Satelite1 & 0.07 & 29.02 \\
\hline Penset & 0.02 & 5.89 \\
\hline Ubitwins & 0.05 & 9.87 \\
\hline hamprhazen & 0.28 & 116.85 \\
\hline moflower & 17.65 & 11366.83 \\
\hline nitktaylor & 0.2 & 667.85 \\
\hline quickie & 1.53 & 20452.38 \\
\hline shadowlane & 0.1 & 33.59 \\
\hline widustob & 0.3 & 690.07 \\
\hline woodswu & 0.63 & 758.36 \\
\hline
\end{tabular}

\section{Conclusion}

Method ImgDataRet has performed well only on standard images like brock-26, keller-5 and quickie-12. The main feature which is taken with serious attention is image preprocessing. In image preprocessing loaded input image undergoes segmentation, finding bright intensity values of pixels and their belongingness to particular region is noted in the form of feature. Out of which extracted $2 \times 1$ matrix is maintained. This matrix shows presence of image object particles in an image. This algorithm is tested on light background images of courtesy standard benchmark images; this has found its moderate performance with these images as compared to other allied approaches like Convolution Based Detection, Hybrid Projection, Neural based and Multi-View Approach. It has also tested over complex image like hamming-200 and MANN-400; it has performed relatively poorer on these kinds of complex images.

\section{References}

[1] A. Jain, (1989), Fundamentals of Digital Image Processing, Prentice-Hall Inc. New Delhi.

[2] Blake and Michael, (2013), Active Contours and Image Particles, Springer

[3] Burger and Lenddins, (2010), Digital Image processing using Matlab, Mcgraw Hill's New Delhi

[4] Dwayne and Philipps, (2000), Analyzing and Enhancing Digital Images, R \& D Books, US

[5] Pettis and Jay, (2012), Getting Started with Image Tools, O'Reilly Media, New Delhi

[6] Hanry and Ford, (2010), Fundamentals of image Processing, Dartmouth Inst Press, US

[7] Edwads, Staples, Thei zNose, (2000) "A New Electronic Nose Using Acoustic Technology", Acoustical Society of America, P.242-250

[8] Koprowsky, Wrobel and Ketty, (2011), Image Processing in Optical Coherence Tomography, University of Silesia, US

[9] Phillips, Gleeson, (2001) "Volatile organic compounds in breath as markers of lung cancer:a cross-sectional study", Lancet , P.353:1930-33

[10]Berg, Olsson, Lindblad, and Chilo, (2008) "Automatic design of pulse coupled neurons for image segmentation," Neurocomputing, vol. 71, nos. 10-12, pp. 1980-1993.

[11]Cao, Luo, Kautz, and Huang, (2009) "Image annotation within the context of personal photo collections using hierarchical event and scene models," IEEE Trans. Multimedia, vol. 11, no. 2, pp. 208-219

[12]Laeng, Okubo, Saneyoshi, Michimata, (2011), "Processing spatial relations with different apertures of attention", Cogn Sci35:297-329

[13]Yufeng Zeng, (2013), Image Fusion and Its Applications, Intech Press, Canada

[14]Lew, Sebe, Djeraba, and Jain, (2006) "Content-based multimedia information retrieval: State of the art and challenges," ACM Trans. Multimedia Comput., Commun. Appl., vol. 2, no. 1, pp. 1-19

[15] Westland S. (2012) "Computational Colour Science using MATLAB 2e".By WILEY ISBN: 978-0-47066569-5 p:27-104.

[16]Wu, Chensheng, Christopher, Davis.(2014) "Modified plenoptic camera for phase and amplitude wavefront sensing." International Society for Optics and Photonics, pp. 88740I-88740I.

[17]Wei, Wang, and Lai, (2012) "Compact image representation model based on both nCRF and reverse control mechanisms," IEEE Trans. Neural Netw. Learn. Syst., vol. 23, no. 1, pp. 150-162.

[18]L.Tadic, (2012) "Color Space Basics", AMIA Tech Review. Issue 4. pno 123

[19] Chen, Park, Ma, and Ala, (2011) "A new automatic parameter setting method of a simplified PCNN for image segmentation," IEEE Trans. Neural Netw., vol. 22, no. 6, pp. 880-892.

[20] Afraz and Cavanagh, (2009)."The gender specific face after effect is based in retinotopic not spatiotopic coordinates across several natural image transformations." $\quad$ J.Vis. $\quad 9,10.1-$ 10.17.doi:10.1167/9.10.10 


\section{International Journal of Science and Research (IJSR)}

ISSN (Online): 2319-7064

Index Copernicus Value (2015): 78.96 | Impact Factor (2015): 6.391

\section{Author Profile}

Gurjinder Singh received BCA in 2012 and Msc (IT) in 2014 Under GNDU (Guru Nanak Dev University, Amritsar).Now His M.Tech(IT) perusing From Guru Kashi University, Talwandi Sabo (Bathinda), India. 